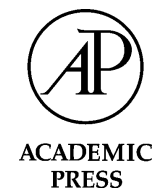

\title{
1D and 2D Fourier-based approaches to numeric curvature estimation and their comparative performance assessment
}

\author{
Leandro Farias Estrozi, ${ }^{a}$ Luiz Gonzaga Rios-Filho, ${ }^{\text {a }}$ Andrea Gomes \\ Campos Bianchi, ${ }^{a}$ Roberto Marcondes Cesar Jr., ${ }^{\mathrm{b}}$ and \\ Luciano da Fontoura Costa ${ }^{\mathrm{a}, *}$ \\ ${ }^{a}$ Cybernetic Vision Research Group, IFSC-USP, Caixa Postal 369, São Carlos, São Paulo, 13560-970, Brazil \\ ${ }^{\mathrm{b}}$ Creative Vision Research Group, Department of Computer Science, IME, University of São Paulo, \\ Rua do Matão, 1010, São Paulo, 05508-900, Brazil
}

\begin{abstract}
A careful comparison of three numeric techniques for estimation of the curvature along spatially quantized contours is reported. Two of the considered techniques are based on the Fourier transform (operating over 1D and 2D signals) and Gaussian regularization required to attenuate the spatial quantization noise. While the 1D approach has been reported before and used in a series of applications, the 2D Fourier transform-based method is reported in this article for the first time. The third approach, based on splines, represents a more traditional alternative. Three classes of parametric curves are investigated: analytical, B-splines, and synthesized in the Fourier domain. Four quantization schemes are considered: grid intersect quantization, square box quantization, a table scanner, and a video camera. The performances of the methods are evaluated in terms of their execution speed, curvature error, and sensitivity to the involved parameters. The third approach resulted the fastest, but implied larger errors; the Fourier methods allowed higher accuracy and were robust to parameter configurations. The $2 \mathrm{D}$ Fourier method provides the curvature values along the whole image, but exhibits interference in some situations. Such results are important not only for characterizing the relative performance of the considered methods, but also for providing practical guidelines for those interested in applying those techniques to real problems.
\end{abstract}

(c) 2002 Elsevier Science (USA). All rights reserved.

\footnotetext{
* Corresponding author.

E-mail addresses: lfestroz@if.sc.usp.br (L.F. Estrozi), lgrios@if.sc.usp.br (L.G. Rios-Filho), campos@if.sc.usp.br (A.G. Campos Bianchi), luciano@if.sc.usp.br (L.F. Costa).
} 
Keywords: Digital signal processing; Curvature estimation; Differential geometry; Numerical methods; Fourier transform; Shape analysis; Performance assessment; Gaussian regularization

\section{Introduction}

The analysis of 2D shapes is one of the most classical, important, and widely studied problems in pattern recognition and computer vision, finding applications in a myriad of practical problems. Nevertheless, in spite of its seeming simplicity, there is no definitive approach to 2D shape analysis and classification, and much effort is still needed mainly in order to assess the existing techniques and to improve the accuracy and recognition rates of the most reliable approaches. Among the different methods for 2D shape analysis, those based on the shape boundary curvature constitute some of the most comprehensive and promising, as discussed in more detail in the following. The main objectives of the present article are twofold: to introduce a new Fourier-based 2D curvature estimation technique and to comparatively evaluate the performance of three numerical methods for digital curvature estimation. Such results can provide valuable insight not only on the specific advantages and disadvantages of each method, but at the same time offer practical guidelines for those interested in applying the techniques in real problems. In addition, the adopted performance assessment framework can be generalized to other curvature estimation techniques.

First, as far as information preservation is concerned, curvature is a complete representation, since the original curve can be reconstructed (up to rigid-body transformations). Furthermore, since it is a well-accepted fact that not all points on shape boundaries are equally relevant, finding the more salient (or critical) points is an extremely important task for feature extraction and contour segmentation. Curvature also plays a central role within this context: there are various methods that search for these critical points in terms of local maximum and minimum curvature points, as well as zero curvature (straight line) portions. The curvature is invariant to rigid-body transformations (i.e., translations, rotations, and reflections). In addition, psychophysical evidences have also shown that curvature is an extremely important cue for our visual perception processes [1], in such a way that curvature peaks tend to correspond to the salient shape points. Finally, interesting physical analogies based on the curvature concept have led to powerful shape analysis techniques. For instance, elasticity theory provides the useful concept of bending energy, a global feature calculated in terms of the curvature along the contour [2,3], which can be used for characterizing shape complexity [4].

This work describes and discusses a performance assessment framework for digital curvature estimation methods, as well as the results of assessing two different approaches based on Fourier properties and a spline-based interpolation scheme [5,6]. The emphasis given in the current article to the Fourier approach is motivated by the inherent high accuracy allowed by this approach [7-10]. In other words, the Fourier approach allows the consideration of the whole neighborhood around each point at which the curvature is to be estimated, not only a limited neighborhood and resolution as implied by several alternative methods such as [5,6]. The discrete Fourier transform can also be effectively executed by using the FFT. Additional bonuses allowed by this approach include the fact that the shape 
can be easily smoothed in the Fourier domain and that the Fourier descriptors, which are valuable resources for the shape characterization and classification, are obtained as byproducts.

Basically, there are two different ways of estimating the curvature of a digital shape, i.e., either from the object parametrized outline or directly from the $2 \mathrm{D}$ image by using the concept of isopotential curves. Contour-based approaches are more popular and widely used in different vision problems such as in biomedicine, fingerprint recognition, and OCR [11-13], presenting several interesting advantages such as the fact that dealing inherently with 1D data representations is computationally less demanding and the existence of substantial technical literature on contour analysis [7].

Nevertheless, one of the main drawbacks of this approach is the fact that the object must have its contour extracted in parametric fashion before further analysis [7]. On the other hand, the latter class of techniques, which is applied directly to the 2D image, does not depend on contour tracking, allowing dedicated hardware to be used to implement the imaging procedures (such as convolution). Furthermore, the size of the input data becomes independent of the object perimeter, which does not hold to the contour-based approach, since the number of points of the extracted contour varies with the object perimeter. A good discussion on the robustness of computing derivatives over a level set can be found in [14].

The reader is referred to [15-18] for a detailed review of the many alternative curvature estimation methods for digital images. The main problem that must be solved for digital curvature estimation is that the curvature expression involves differentiation of discrete data, which is an ill-posed problem that must be circumvented by the introduction of a regularization procedure. The standard approach is based on smoothing the data, e.g., by convolving the contour with a gaussian kernel, which is the case, for instance, in the now classical work of Mokhtarian and Mackworth [19]. Alternative approaches include local interpolation of the data [6] and the use of different discrete curvature measures, such as the $c$-curvature of Davis [20]. On the other hand, the 2D-based techniques have also received attention from the image processing community. Some examples of works devoted to this approach can be found in [21,22].

Another important aspect addressed in the present work concerns the validation and comparison of the 1D and 2D Fourier-based approaches. Indeed, vision science researchers have been criticized not only for not spending enough attention on characterizing the performance of new approaches respectively to a representative set of data, but also for not comparing such new techniques with a more traditional alternative [23,24]. Only more recently have some works addressed the topic of performance assessment in a systematic way [25-28]. One of the main characteristics of the present work is to go a long way toward addressing these criticisms. First, special care is spent in trying to define a representative set of data (see Section 3.1), with special attention given to 2D closed, simple (in the sense of being Jordan curves) parametric curves. The reason for concentrating on closed curves is that this type of curve is inherently compatible with Fourier-based derivative estimation. In other words, the fact that both these curves and the discrete Fourier transform are periodical allows continuity of the curve and its derivatives, which is not generally verified for open curves.

While it should be stressed at the outset that it is virtually impossible to consider a wholly representative set of testing data, it is believed that it is better to consider some 
large, even if not completely representative, set of shapes than to consider just a handful, as sometimes happens in the related literature. More specifically, three kinds of curves have been considered: (a) those defined by analytical expressions, such as sinusoidal circles and spirals; (b) curves defined by B-splines [5]; and (c) curves synthesized in terms of spectral content [29]. It is important to note that, in each of these cases, it is possible to obtain an analytical and absolutely precise quantification of the point curvature along the contours (see Section 3.2), to be used as a standard for comparison. Each of these is spatially sampled, before numeric estimation, according to four quantization schemes: (i) gridintersect quantization, (ii) square box quantization, (iii) using a conventional camera, and (iv) using a conventional scanner. Observe that (iii) and (iv) also involve printing the shapes through a conventional laser printer. These two latter schemes provide a more global characterization of the considered methods given typical practical situations.

Great attention has also been focused in defining unbiased and comprehensive merit figures allowing proper characterization of the considered techniques (see Section 3.3). The important features to be considered included the accuracy in the curvature calculation, defined in terms of root mean square (RMS) error between the analytical and numerically estimated curvatures, the robustness to parameter tuning, expressed in terms of the distribution of the optimal parameters (the scale parameters defined by the standard deviation of the smoothing Gaussians), and the execution time. A more detailed discussion of such measures can be found in Section 4.

In addition to trying to characterize the performance of the considered techniques according to a formal, comprehensive, and comparative fashion, there are some particularly interesting questions, inherently defined by the specific features of the Fourier techniques, which should at least partially be answered by the considered framework. For instance, since both first and second order derivatives are needed in the 1D and 2D approaches, it would be interesting to verify the use of two distinct standard deviation values in the regularizing Gaussian smoothing. In other words, since the second order derivatives imply higher enhancement of high frequencies than those needed for first order derivatives, it is interesting to check whether the use of a larger smoothing for the second derivative would lead to improved accuracy. Another important point to be investigated concerns the robustness of the techniques given different parameter settings.

The current article starts by describing the three curvature estimation numerical approaches considered, covering the 2D Fourier-based approach (first outlined in [30]) in more detail. Then, the performance assessment framework is presented in detail, which is followed by the obtained results and the respective discussion. The paper concludes by presenting the overall conclusions as well as possibilities for future developments.

\section{Fourier-based curvature estimation}

As is well known from linear algebra, unitary linear transformations provide a complete (in the sense of being invertible) alternative representation of the signals in the "time" (1D signals) or "spatial" (2D signals) domains. Indeed, the net effect of such transformations consists in expressing the signal in a new basis, which is obtained by rotating the coordinate system. The advantage of transformations such as the Fourier transform, which are quasi- 
unitary, lies in the fact that the complex exponential basis used in this transformation, because of its highly correlated nature, usually allows the signal to be represented in a much more compact form, i.e., with just a few spectral components. However, the Fourier transform also possesses a series of additional interesting properties, such as the differentiation property, given by Eqs. (1) and (2), respectively, to 1D and 2D signals, where $H(f)=\Im(h(t))$ and $H(u, v)=\Im(h(x, y))$. Such properties allow an interesting means for numeric estimation of derivatives, since the Fourier transform can be fast and effectively performed numerically

$$
\begin{aligned}
& \frac{d^{n}}{d t^{n}} h(t)=\Im^{-1}\left\{(i 2 \pi f)^{n} H(f)\right\}, \\
& \frac{\partial^{n}}{\partial x^{n}} \frac{\partial^{m}}{\partial y^{m}} h(x, y)=\Im^{-1}\left\{(i 2 \pi u)^{n}(i 2 \pi v)^{m} H(u, v)\right\},
\end{aligned}
$$

where $i=\sqrt{-1}$. Given that curvature has a differential nature, as it is clear from Eq. (3), it is in principle possible to use the properties (1) and (2) as a means for numerical estimation of curvature. Observe that the $x$ and $y$ variables in Eq. (3) refer to the parametric functions $x(t)$ and $y(t)$ defined by the contour. Initially proposed in [31], in terms of Fourier series and Kaiser regularizing windows, this possibility has been more extensively developed and applied in a more recent series of developments [7-9] and which rely on the Fourier transform and Gaussian smoothing, being essential for regularizing the high frequency noise introduced by the spatial quantization. Indeed, the standard deviation of the regularizing Gaussian defines a scale parameter allowing multiresolution representation of the estimated curvature [8,9]. While such works focused curvature estimation of $2 \mathrm{D}$ closed contours, done in terms of 1D Fourier transform, it is also interesting to investigate the possibility of using 2D Fourier transform. The main advantage of such an approach is that it can be applied to estimate the curvature, by using Eq. (4), along all the isopotential curves defined by a surface $\phi(x, y)$ containing the original shape contour as one of its level curves, which can be done. A secondary advantage of such an approach is that the size of the input data does not necessarily vary with the perimeter of the shapes, as happens in the $1 \mathrm{D}$ approach.

$$
\begin{aligned}
k & =\frac{\dot{x} \ddot{y}-\dot{y} \ddot{x}}{\left(\dot{x}^{2}+\dot{y}^{2}\right)^{3 / 2}}, \\
k & =\nabla \cdot \frac{\nabla \phi}{\|\nabla \phi\|}=\frac{\phi_{x x} \phi_{y}^{2}-2 \phi_{x} \phi_{y} \phi_{x y}+\phi_{y y} \phi_{x}^{2}}{\left(\phi_{x}^{2}+\phi_{y}^{2}\right)^{3 / 2}} .
\end{aligned}
$$

The next sections present the 1D and 2D Fourier-based curvature estimation techniques, respectively, with special attention given to the latter, since it is presented here for the first time.

\subsection{Curvature estimation based on parametric curve approximation}

This first approach assumes that the derivatives of the curvature expression, Eq. (3), are calculated based on a local piecewise approximation of the contour points. More specifically, suppose that the curve to be adjusted is a cubic parametric polynomial in $t$, 
with $t \in[0,1]$, which approximates a curve segment between two contour points $A(t=0)$ and $B(t=1)$. Therefore, for this curve segment, the approximation curve is defined as:

$$
\begin{aligned}
& x(t)=a_{1} t^{3}+b_{1} t^{2}+c_{1} t+d_{1}, \\
& y(t)=a_{2} t^{3}+b_{2} t^{2}+c_{2} t+d_{2} .
\end{aligned}
$$

By calculating the respective derivatives and substituting them in the curvature expression, Eq. (3), it can be easily verified that the curvature at the point $A(t=0)$ of the contour is

$$
k=2 \frac{c_{1} b_{2}-c_{2} b_{1}}{\left(c_{1}^{2}+c_{2}^{2}\right)^{3 / 2}} .
$$

Medioni and Yasumoto [6] have used cubic B-splines with equally spaced nodes for piecewisely adjusting the parametric curve segments. The coefficients $b_{1}, b_{2}, c_{1}$, and $c_{2}$ of Eq. (7) above can be calculated, from the above expressions, as (refer to [6] for further detail):

$$
\begin{aligned}
& b_{1}=\frac{1}{12}\left(\left(x_{n-2}+x_{i+2}\right)+2\left(x_{n-1}+x_{n+1}\right)-6 x_{n}\right), \\
& b_{2}=\frac{1}{12}\left(\left(y_{n-2}+y_{n+2}\right)+2\left(y_{n-1}+y_{n+1}\right)-y_{n}\right), \\
& c_{1}=\frac{1}{12}\left(\left(x_{n+2}-x_{n-2}\right)+4\left(x_{n+1}+x_{n-1}\right)\right), \\
& c_{2}=\frac{1}{12}\left(\left(y_{n+2}-y_{n-2}\right)+4\left(y_{n+1}+y_{n i-1}\right)\right) .
\end{aligned}
$$

The curvature is calculated by substituting the above coefficients in the curvature equation (7).

\subsection{ID curvature estimation based on the fourier derivative property}

The curvature estimation method discussed in this section originates from the curvegram concept discussed in [8,9] and has been considered in several applications $[4,7,10]$. Let $c(n)=(x(n), y(n))$ be the parametric contour of interest, with $n=0, \ldots, N-1$, and let $N$ be the number of points along the boundary. The contour can be represented as a complex signal $u(n)=x(n)+i y(n)$. A fundamental tool for this approach is the 1D Fourier transform pair of $u(n)$, given by

$$
\begin{aligned}
& U(s)=\Im\{u(n)\}=\sum_{n=0}^{N-1} u(n) \mathrm{e}^{-i 2 \pi(s n / N)}, \quad s=0, \ldots, N-1, \\
& u(n)=\Im^{-1}\{U(s)\}=\frac{1}{N} \sum_{s=0}^{N-1} U(s) \mathrm{e}^{i 2 \pi(s n / N)} .
\end{aligned}
$$

The auxiliary function $\eta(s)$ is a useful tool for the estimation of the discrete derivatives of $u(n)$,

$$
\eta(s)= \begin{cases}s, & \text { if } s=0,1, \ldots,(N-\text { floor }(N / 2)-1), \\ N-s, & \text { if } s=(N-\text { floor }(N / 2)),(N-\text { floor }(N / 2)+1), \ldots,(N-1),\end{cases}
$$


with floor $(N / 2)$ being the truncation function. Function $\eta(s)$ implements the necessary alignment because of the representation normally produced by the DFT (i.e., the frequency representation formed by the second part followed by the first part of the next period). The signals should be smoothed because of a high frequency enhancement effect produced by numerical differentiation, which is achieved by taking the smoothed version $u(n, a)$ of $u(n)$,

$$
u(n, a)=\Im^{-1}\left\{U(s) G_{a}(s)\right\},
$$

where $G_{a}(s)=\exp \left(-(a \eta(s))^{2}\right)$. Contour shrinking is avoided by taking for the spatial scale parameter $a$ a value as small as possible in order to filter the spatial quantization noise and not distort the contour too much. The smoothed first and the second derivatives of $u(n)$ are defined as

$$
\begin{aligned}
& \dot{u}(n, a)=\Im^{-1}\left\{i 2 \pi \eta(\mathrm{s}) U(s) G_{a}(s)\right\}, \\
& \ddot{u}(n, a)=\Im^{-1}\left\{-(2 \pi \eta(\mathrm{s}))^{2} U(s) G_{a}(s)\right\} .
\end{aligned}
$$

The multiscale curvature description of $u(n)$ is given by

$$
k(n, a)=\frac{-\Im\left\{\dot{u}(n, a) \ddot{u}^{*}(n, a)\right\}}{|\dot{u}(n, a)|^{3}},
$$

where $z^{*}$ denotes the complex conjugate and $|z|$ denotes the complex modulus of $z$.

Although it is possible to apply the 1D Fourier method to contours with any amount of points, it is often much more efficient to consider the number of points which are integer powers of two, because this situation allows for fast Fourier transforms. This can be easily accomplished by linearly interpolating the parametric curves along evenly distributed portions of the original curve, in order to produce a new representation with the desired amount of points, i.e., the smallest integer power of two larger than the original number of points.

\subsection{D Fourier-based method}

Given a regular and simple curve $c(t)$, not necessarily represented in parametric fashion, it has to be in some way extended onto the $2 \mathrm{D}$ domain before the $2 \mathrm{D}$ differential operators can be applied. Two possible alternatives for implementing such an embedding are (i) to fill the interior of $c(t)$ with 1 , assign $\frac{1}{2}$ to the contour elements, and zero to the background; and (ii) to apply a signed distance transform to the contour in such a way that the interior becomes negative (resp. positive) and the exterior positive (resp. negative). Three issues should be taken into account while choosing between such schemes. First, as far as execution time is concerned, alternative (i) is simpler and therefore faster. Second, the larger the spread of the extended surface, the higher the interference between parametrically distant but geometrically close portions of the curve. In this respect, scheme (i) is again more suitable. Third, smoother extended surfaces tend to allow fewer discontinuities while differentiating in order to estimate curvature. While the second strategy is in principle more likely to induce smoother extensions, the Gaussian low-pass filtering inherent to the Fourier approaches, provided a suitable standard deviation is used, 
can also smooth the more abrupt transition implied by scheme (i). In order to favor speed and minimize curve interferences, the present article has adopted the former scheme. Once such an extension $\phi(x, y)$ is achieved, the curvature of the contour defined by $\phi(x, y)=a$ (a level-curve) can be estimated by using Eq. (4) implemented in the Fourier domain considering Eq. (2).

As with the 1D approach described in the previous section, it is necessary to regularize $\phi(x, y)$, since it is represented in a spatially sampled space. This will be done by convolving $\phi(x, y)$ with a circularly symmetric 2D Gaussian given by Eq. (19), which is more effectively done in the Fourier domain,

$$
G_{\sigma}(x, y)=\frac{1}{2 \pi \sigma^{2}} e^{-\left(x^{2}+y^{2}\right) / 2 \sigma^{2}} .
$$

It should be observed that, though initially all the original contour points lie at the same level-curve, this is no longer true after the regularization. Since for small smoothing degree the curves do not shift too much, the curvatures are henceforth taken at the original coordinates.

\section{The evaluation framework}

In order for different numerical methods to be properly compared, it is important to define an overall computational framework which is as fair and comprehensive as possible. This endeavor entails three main issues, namely defining a suitable set of test shapes, modeling the spatial quantization schemes, and identifying suitable merit figures which can express how the methods fulfill the principal properties expected of them. The following sections present and discuss each of these issues, respectively.

\subsection{The considered shapes}

Before the performance of different curvature estimation techniques can be properly evaluated, it is necessary to define the basic standard input for the methods. Three important properties are expected from such shapes. First, they must allow an analytic description of curvature, since this is essential for quantifying the accuracy of each considered method. Second, the set of shapes must be as general as possible in order to provide a representative sample of the shapes to be found in typical shape analysis applications. While the former of these features does not pose a real problem in practice, it is virtually impossible to consider a fully representative set of shapes, since the possible objects in nature define a virtually unlimited number of shapes. The limiting factor here is the computational resources demanded to process and analyze the performance respectively to a very large set of shapes. The third requirement concerns the fact that the analytical curvature, defined by the curves, must not be too high and not vary too abruptly, in order to be properly represented in the orthogonal grid used for spatial quantization of the curves, since it is not fair to expect a numerical technique to estimate a curvature which is not properly represented in the image. This requirement has been implemented by not using any curve implying curves whose radius of curvature is smaller than $\frac{1}{3}$ of a pixel, except for a small number of small isolated regions. 


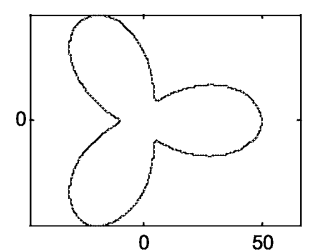

(a)

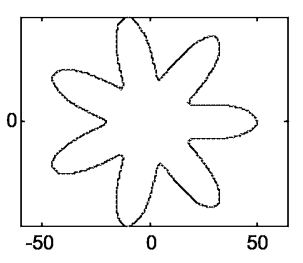

(b)

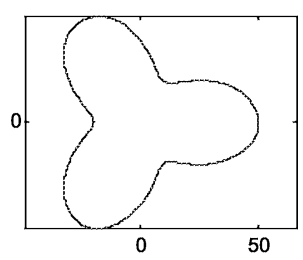

(c)

Fig. 1. Sinusoidal circles with varying number of branches and internal radiuses.

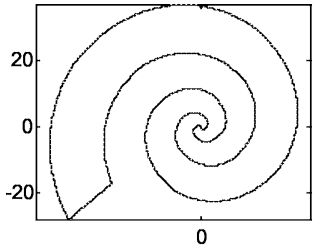

(a)

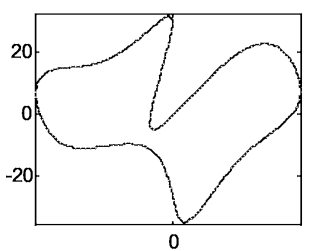

(a)

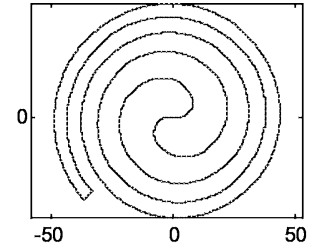

(b)

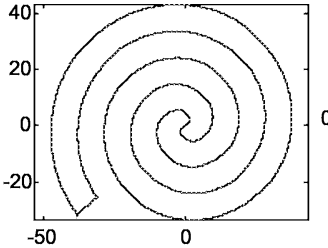

(c)

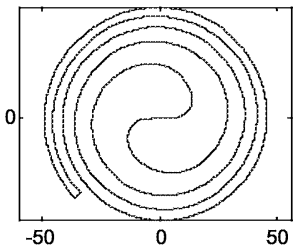

(d)

Fig. 2. Spirals with different width evolutions.

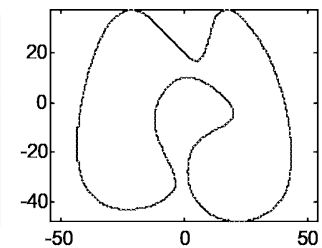

(b)

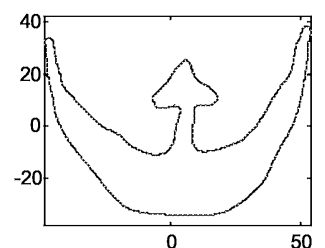

(c)

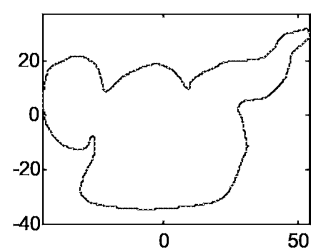

(d)

Fig. 3. Some of the considered B-spline-generated contours.

In order to address the above issues, the current approach has considered three distinct classes of closed, simple (Jordan) curves. First, as illustrated in Figs. 1 and 2, we have analytical curves such as sinusoidal circles and spirals, respectively (though not closed in their analytical versions, these spirals have been closed by hand), which can provide a broad variety of shapes, though being limited by the nature of the respective functions (e.g., harmonic functions will tend to imply smooth curves, and so on). Second, cubic splines generated in terms of control points interactively supplied by the operator, shown in Fig. 3, have been considered in an attempt to represent more general shapes such as those found in natural objects. However, the generality of such splines is also limited by their inherent characteristics, such as their $C^{n}$ parametric continuity. The third class of shapes, presented in Fig. 4, includes curves defined in the Fourier domain [29] and synthesized by the inverse Fourier transform, but again this approach implies some specific properties, such as being $C^{n}$. 


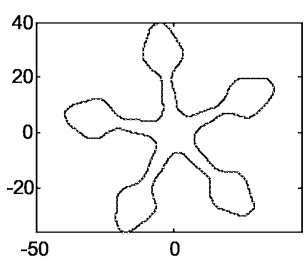

(a)

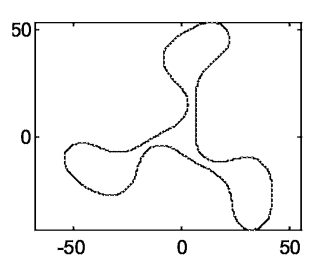

(b)

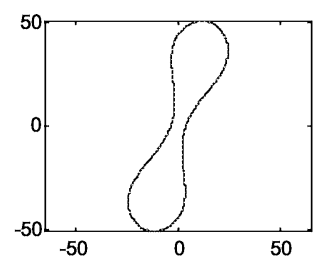

(c)

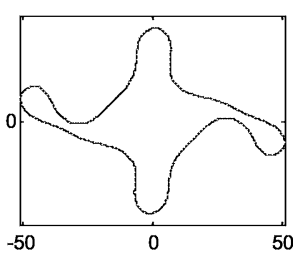

(d)

Fig. 4. Fourier synthesized shapes with different periods and harmonic compositions.

\subsection{Spatial quantization schemes}

Considering that the selected shapes have to be continuous in order to allow exact curvature quantification to be used as a comparison standard, it is necessary to use some suitable spatial quantization method, which maps such continuous curves into the orthogonal grid inherent to any digital image. Four distinct traditional quantization schemes have been considered in the present work in order to provide a general view of the performance of the numerical techniques under varying quantization conditions. Out of these four methods, two are precisely defined in mathematical terms: the grid intersect quantization [32], GIQ, and the square box quantization [32], SBQ. The other two quantization schemes consist of using a standard table scanner (HP Scanjet 4L) and a video camera (Sony CCD IRIS) in order to acquire the images of high quality printouts of the shapes (HP LaserJet 4L, 300 dpi). Figure 5 illustrates the GIQ (a) and SBQ (b) representations of a same shape, and zoomed respective sections (c) and (d). Since the SBQ typically implies a more dense spatial quantization of the original analytical curve, it could be expected that smaller errors would be obtained by a numerical curvature estimation technique operating over such representations.

It should also be observed that the GIQ and SBQ schemes can produce representations including double points, such as that marked with an $*$ in Fig. 6a. Since such double points can make the curve not regular during curvature estimation, it is important to remove them, which is currently done by incorporating additional conditions in the curve quantization.

Special remarks regarding the detection of the shape edges include the fact that, while this operation is not required when considering the GIQ and SBQ schemes, a simple threshold operation followed by binary edge detection is considered for the shapes obtained from the scanner and camera. In practical general situations, traditional edge detection schemes (see, for instance, $[7,33,34]$ ) can be applied. The slightly different edges obtained by such different methods are uniformized by the Gaussian smoothing inherent to the application of different contour extraction algorithms that generally affect curvature estimation [35].

\subsection{Merit figures}

The evaluation framework adopted in the present work consists in presenting the previously mentioned four spatially sampled versions of each of the considered reference shapes 


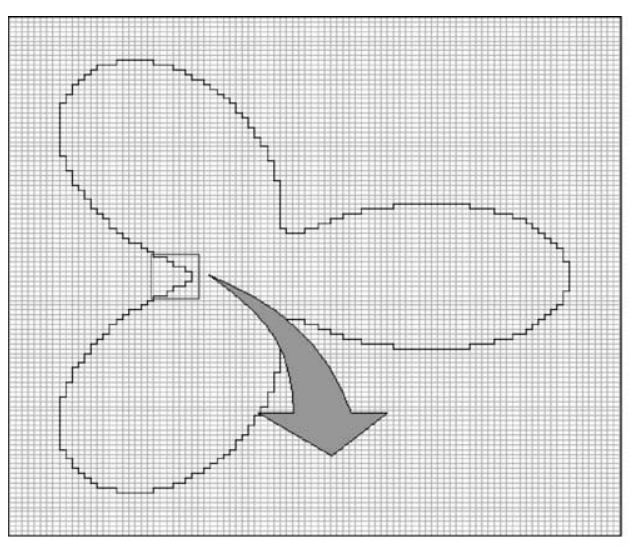

(a)

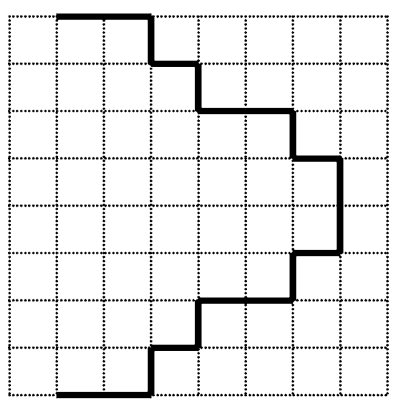

(c)

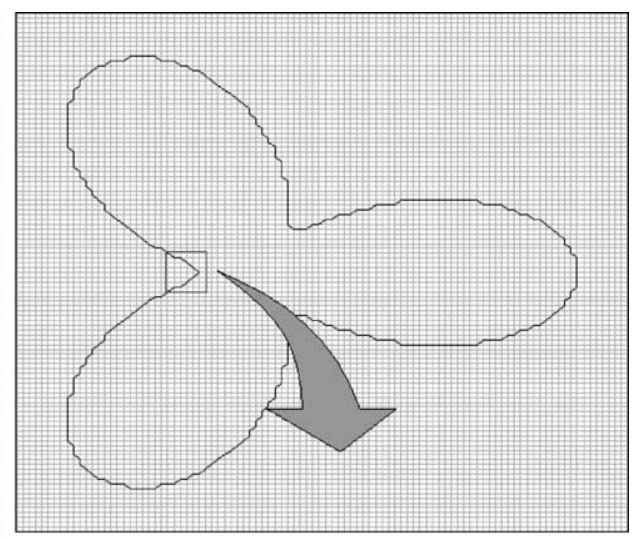

(b)

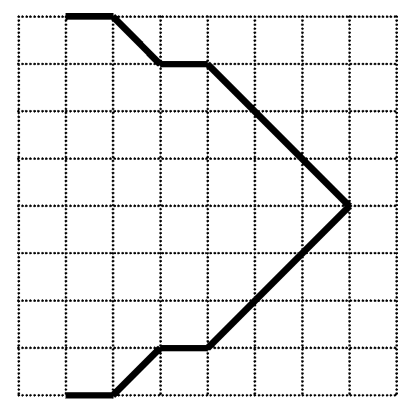

(d)

Fig. 5. Square box (a) and grid intersect (b) quantizations of a specific parametric curve, and respective zoomed sections (c) and (d).

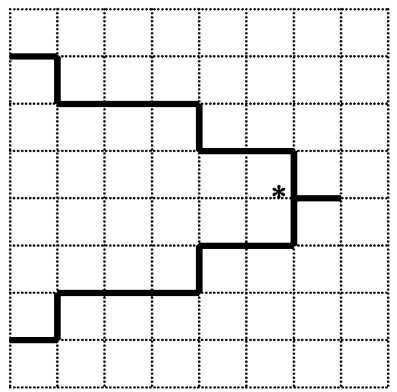

(a)

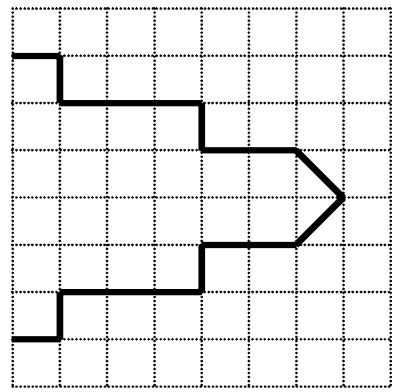

(b)

Fig. 6. Repeated points (a) and representation after removal of such points (b). 
to each numerical method and comparing the analytical and the numerically estimated curvatures along the curve. As already observed, three performance characteristics have been specially taken into account: (a) the implied execution times, (b) the estimation errors, and (c) the sensitivity of the techniques given specific parameter configurations, here quantified in terms of the dispersion of the optimal scale parameter values and the extension of the spatial scales for which the error does not exceed $10 \%$ of the minimal error.

One of the most direct and intuitive ways of quantifying the estimation error is in terms of some traditional metrics, such as the RMS error, an approach that is adopted in the present article. More specifically, for each shape and considered method, a global error $\varepsilon$ is obtained by using Eq. (20), where $k_{o}$ is the calculated curvature using the proposed techniques, $k_{a}$ is analytical quantized curvature, and $N$ is the number of contour points.

$$
\varepsilon_{\mathrm{rms}}=\sqrt{\frac{1}{N} \sum_{i=1}^{N}\left(k_{o}(i)-k_{a}(i)\right)^{2}} .
$$

However, while the Euclidean distance does provide a global measurement of similarity between all the original and estimated curvatures along the curves, it also presents some shortcomings. For instance, a large difference in just a single point may generally influence the overall error. Since such problems will be implied by virtually any alternative metric, we tried to control such effects by having the curvatures (both analytical and numerical) equalized through a sigmoid function (thus limiting curvature values within the $[-3,+3]$ range) before the Euclidean distance is calculated. This process involves Eq. (21), where $x$ denotes a curvature value and $a$ is the maximum allowed curvature absolute value.

$$
S(x)=a\left[\frac{e^{x / a}-e^{-x / a}}{e^{x / a}+e^{-x / a}}\right]=a\left[1-\frac{2}{e^{2 x / a}+1}\right] .
$$

In order to provide a more complete characterization of the estimation error, the Euclidean distances have been organized in terms of histograms.

Concerning the third performance issue, namely the sensitivity of each method given specific parameter configurations, two measures have been considered. First, it is interesting to quantify the dispersion of the optimal parameters. For each estimation technique the best parameter (i.e., the standard deviation) value, in the sense of smaller estimation error, is identified by using a brute force approach (i.e., scanning along the parameter space), and the respective distribution is expressed in terms of respective histograms. Large dispersion values indicate that the optimal standard deviation values vary considerably from one curve to another, making the choice of a suitable parameter particularly difficult; smaller dispersions indicate that the best parameters will be all comprised within a reasonably small interval. The second sensitivity measurement expresses the extension of the spatial scales for which the error does not exceed $10 \%$ of the minimal error in each case (see Fig. 7), here considered in terms of histograms. Consequently, while better performances are characterized by larger extensions, smaller extensions imply high sensitivity to parameter configuration. 


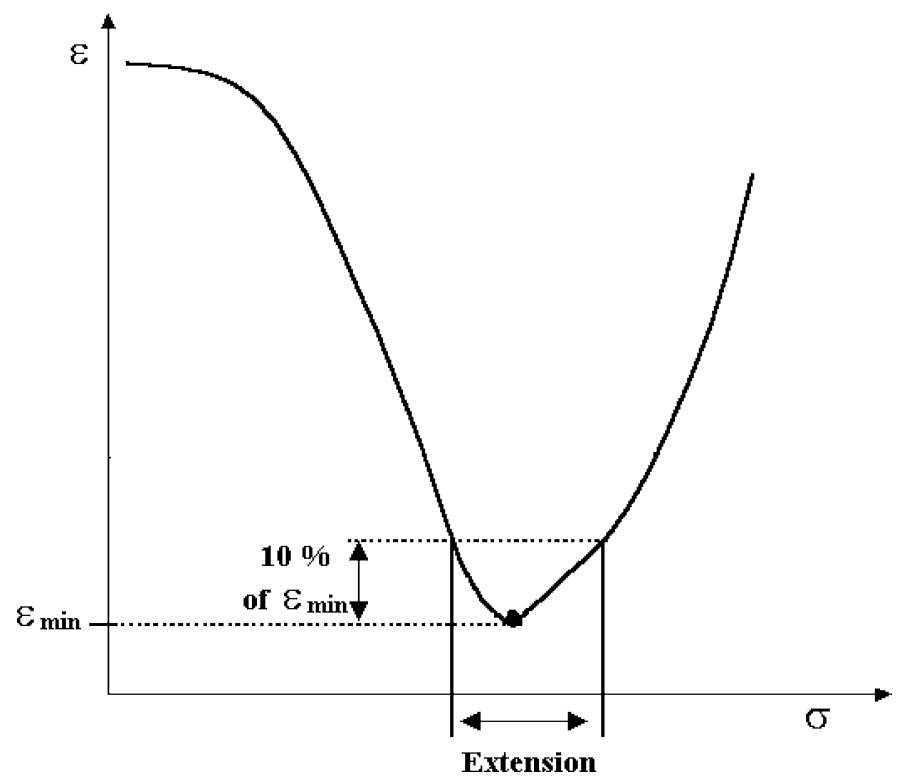

Fig. 7. Spatial scale extension considered as a sensitivity quantitative measure.

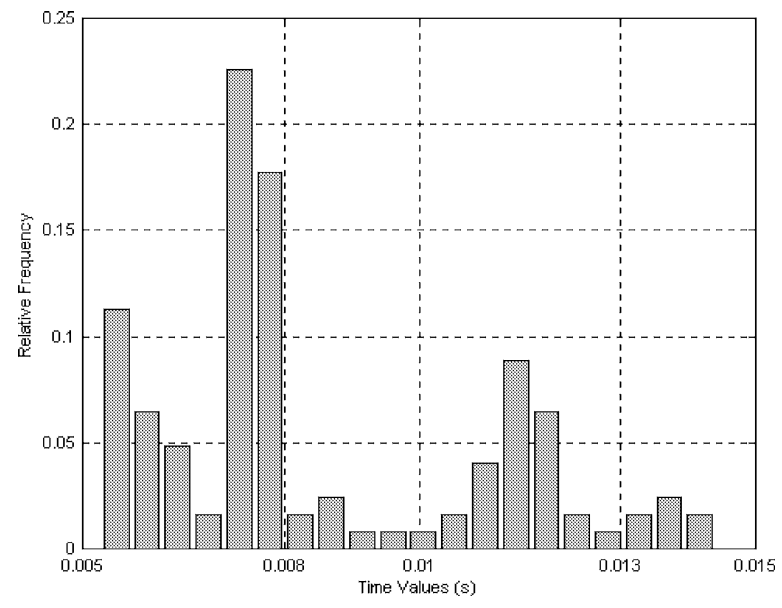

Fig. 8. Time histogram for interpolated version of the 1D Fourier method. Average value (standard deviation $)=0.008( \pm 0.003) \mathrm{s}$.

\section{Results}

The following sections present the performance assessment of the considered numerical curvature estimation techniques with respect to the three considered aspects: (a) execution time, (b) estimation errors, and (c) parameter sensitivity. The henceforth presented results 


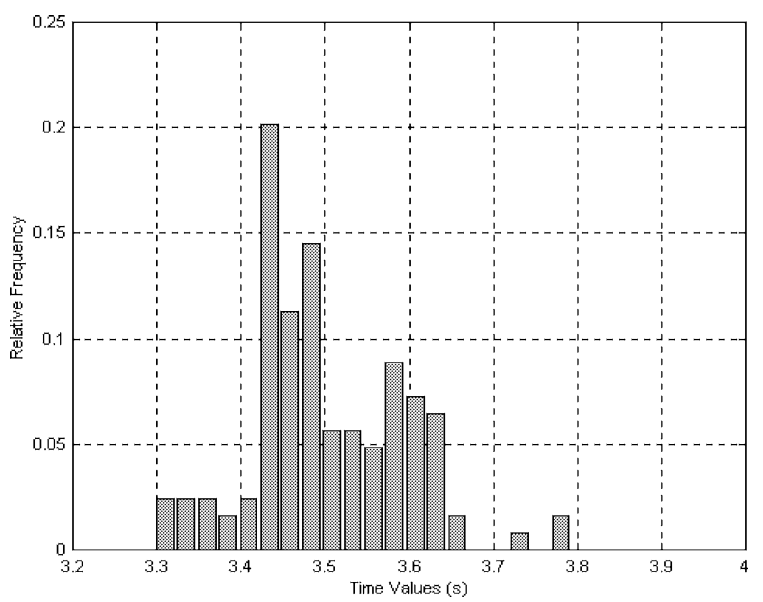

Fig. 9. Time histogram for the 2D Fourier method. Average value (standard deviation) $=3.5( \pm 0.2) \mathrm{s}$.

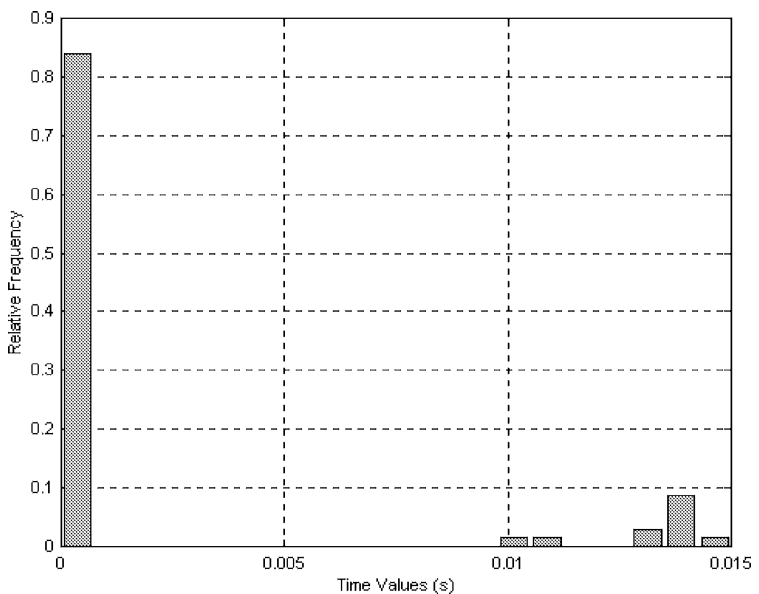

Fig. 10. Time histogram for Medioni and Yasumoto's method. Average value (standard deviation) = $0.002( \pm 0.005) \mathrm{s}$.

were obtained by running Delphi (versions 3 and 4) implementations of the techniques in a Pentium II $333 \mathrm{MHz}, 256$ Mbytes of memory, IBM-PC compatible microcomputer under Windows 95.

\subsection{Execution times}

Histograms of the execution times, obtained by using the operating system internal clock, are presented in Figs. 8-10, to 1D Fourier, 2D Fourier, and Medioni and Yasumoto's approaches, respectively. It should be observed that the times for 1D Fourier are respective to interpolated versions of the curves obtained as described in Section 2.2, since this 


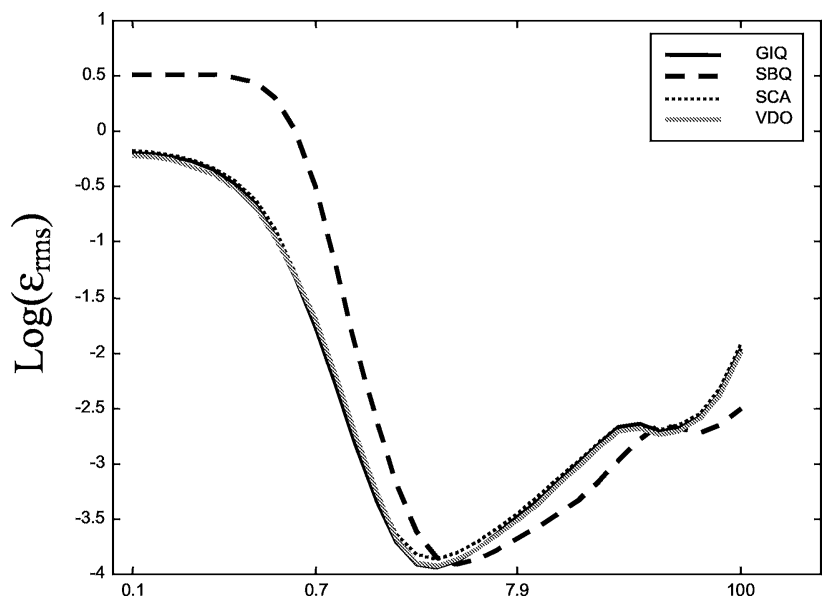

(a)

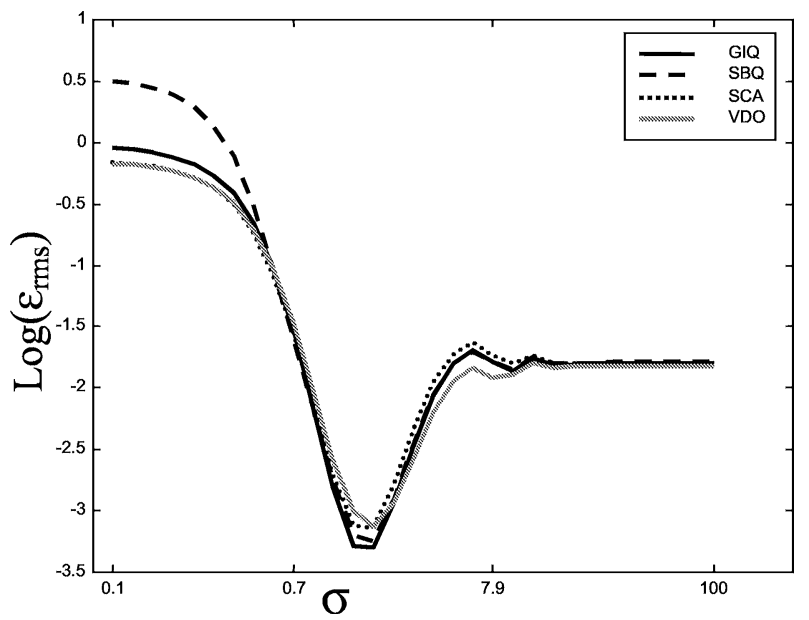

(b)

Fig. 11. Typical behavior of the estimation error for 1D (a) and 2D (b) Fourier-based methods. Both scales are logarithmic.

strategy allows the fastest execution speeds through the FFT. It should be observed that the presented histograms include all the considered curve classes and quantizations. The time required to linearly interpolate the curves in the 1D Fourier approach (explained in Section 2.2) has not been considered in the execution times. When interpolated, the great majority of the curves resulted in 1024 points.

\subsection{Histograms of curvature estimation errors}

Figure 11 presents the typical behavior observed for the estimation error, in terms of the scale parameters (Gaussian standard deviation $\sigma$ ), with respect to 1D and 2D Fourier methods. It should be observed that both scales in such figures are logarithmic. In order 


\section{$\log \left(\varepsilon_{\mathrm{rms}}\right)$}

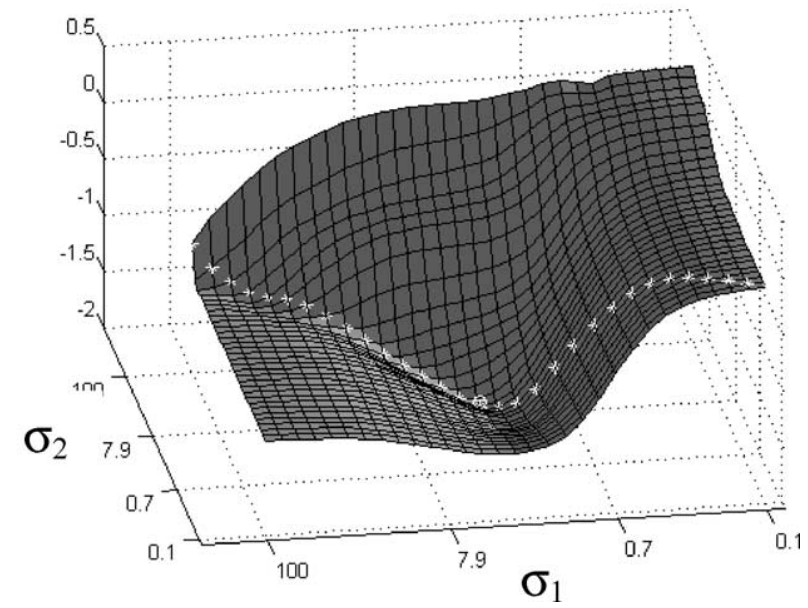

(a)

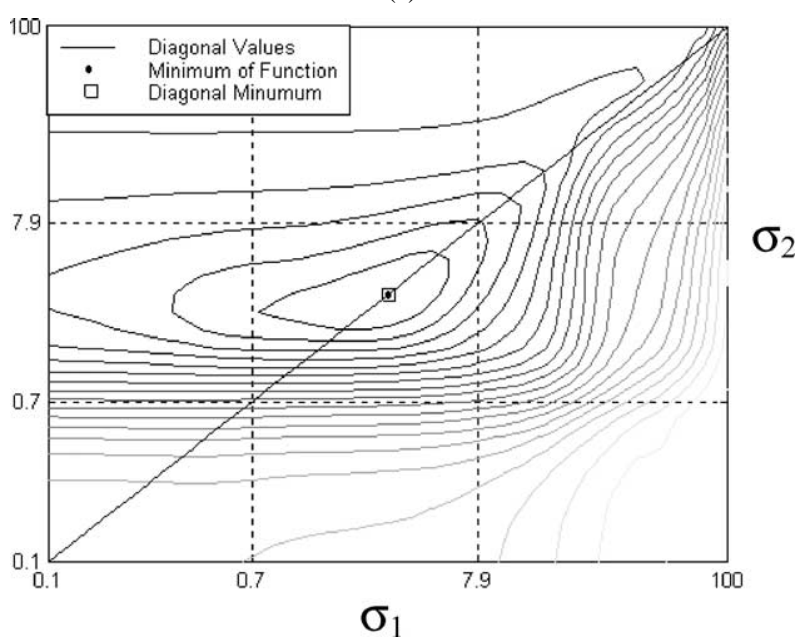

(b)

Fig. 12. Typical errors obtained for curvature estimation considering a series of combinations of the regularizing parameters $\sigma_{1}$ and $\sigma_{2}$. Situation where the minimal error lies on the main diagonal.

to verify the possible improvements allowed by using two distinct values of standard deviations, $\sigma_{1}$ and $\sigma_{2}$, respectively, as regularizing parameters for the first and second derivatives (see Section 1), the Fourier-based methods were run for several combinations of such scale parameters. Figures 12 and 13 present typical results, with respect to the Bspline in Figs. $3 \mathrm{~b}$ and 1c. These two figures illustrate the situations where the minimum error lies on and off the main diagonal.

Since it was verified that distinct values of the standard deviations do not significantly contribute to minimizing the errors, all the subsequent results are respective to a single 


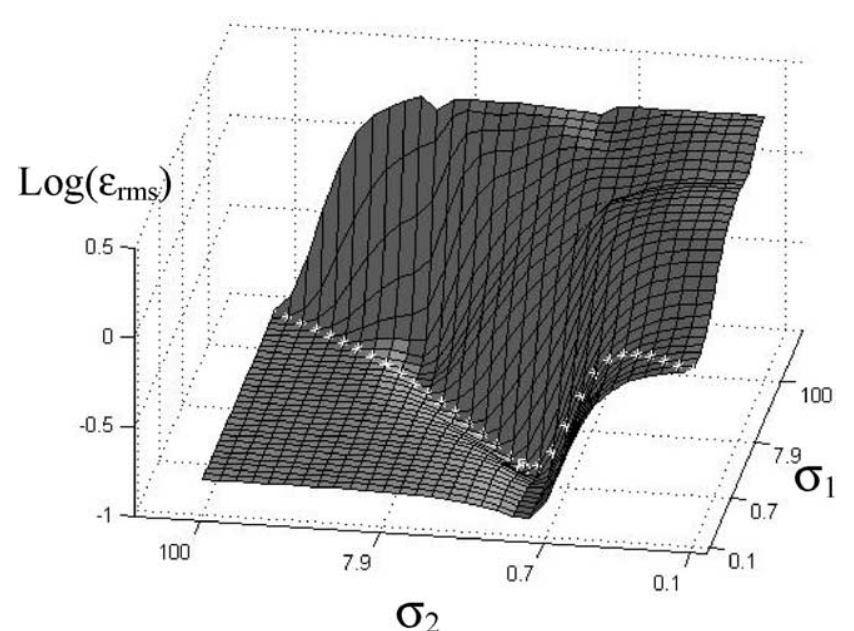

(a)

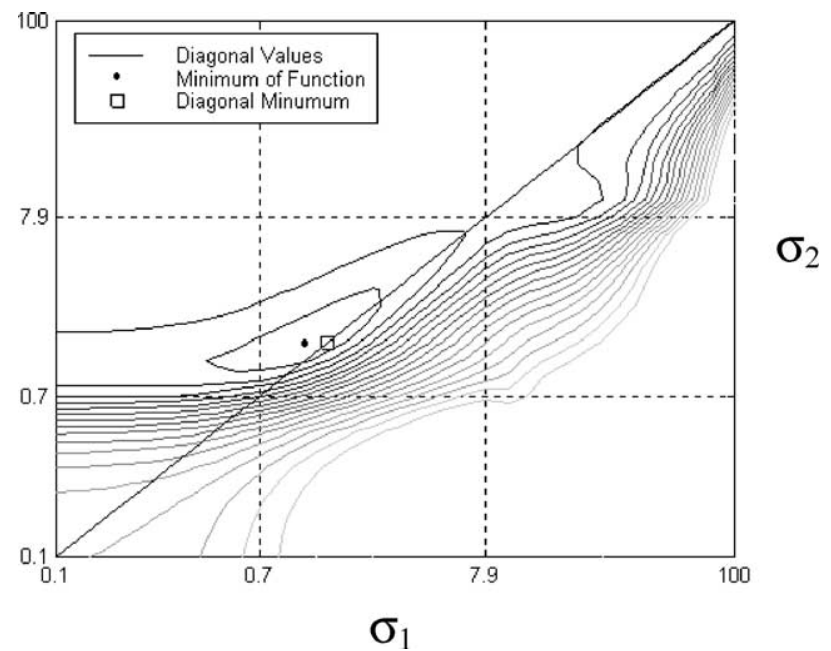

(b)

Fig. 13. Typical errors obtained for curvature estimation considering a series of combinations of the regularizing parameters $\sigma_{1}$ and $\sigma_{2}$. Situation where the minimal error lies off the main diagonal.

standard deviation; i.e., $\sigma_{1}=\sigma_{2}$. Figures $14-16$ present the histograms of errors with respect to the considered three numerical curvature estimation techniques, including all the curves and quantization methods. Figure 14 was obtained for the errors produced by the 1D Fourier-based technique without the interpolation scheme proposed in Section 2.2, in order to allow a comparison between the distinct quantizations. Figures 15 and 16 are respective to the 2D Fourier-based and Medioni and Yasumoto's approaches. Figure 17 presents the errors for Medioni and Yasumoto's approach with respect to the several quantization schemes. 


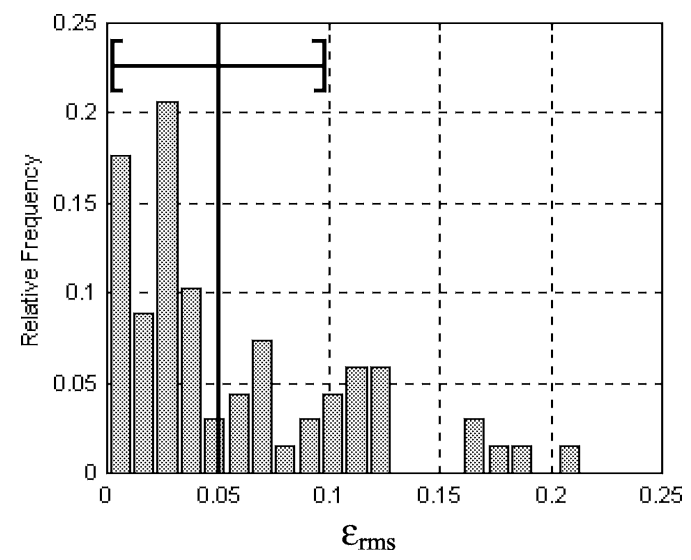

Fig. 14. Curvature estimation errors for the 1D Fourier-based curvature technique, without interpolation. Average value (standard deviation) $=0.05( \pm 0.04)$.

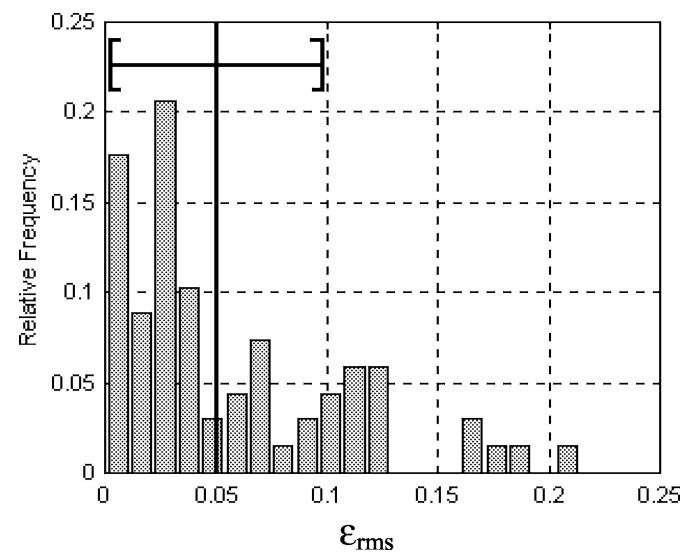

Fig. 15. Curvature estimation errors for the 2D Fourier-based curvature technique. Average value (standard deviation $)=0.05( \pm 0.05)$

\subsection{Parameter sensitivity}

This section presents the results obtained for the quantification of the parameter sensitivity, which is only applicable to the 1D and 2D Fourier-based approaches (Medioni and Yasumoto's technique does not involve any parameters). Figures 18 and 19 presents histograms characterizing the dispersion of the scale parameters corresponding to the minimal curvature estimation errors, with respect to the 1D and 2D Fourier methods. Figures 20 and 21 present the histograms of the extension of the spatial scale ensuring error not larger than $10 \%$ of the minimal error, with respect to the 1D and 2D Fourier techniques. Figure 22 illustrates an interesting observed phenomenon for some curves (especially those exhibit "bottlenecks") corresponding to error peaks caused by interference between 


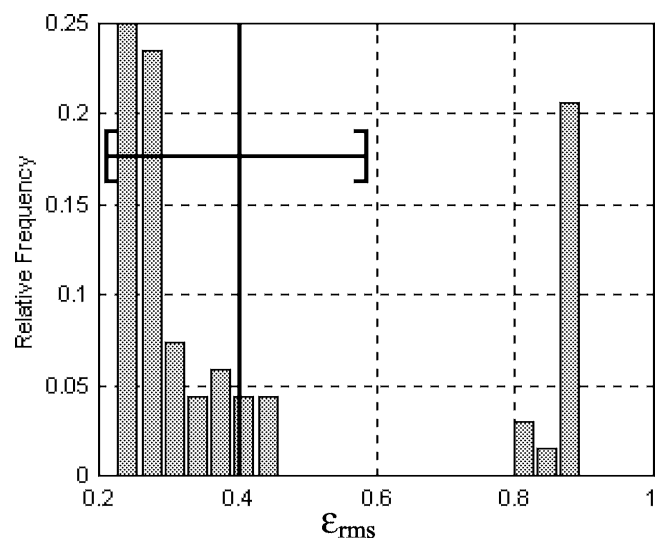

Fig. 16. Curvature estimation errors for Medioni and Yasumoto's approach. Average value (standard deviation $)=0.4( \pm 0.2)$.

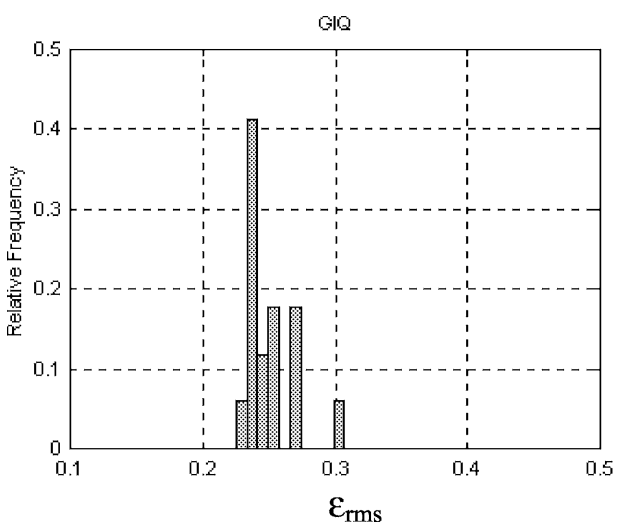

(a)

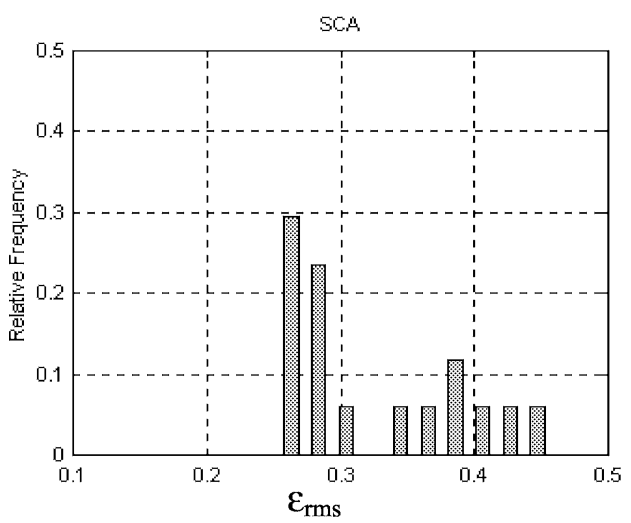

(c)

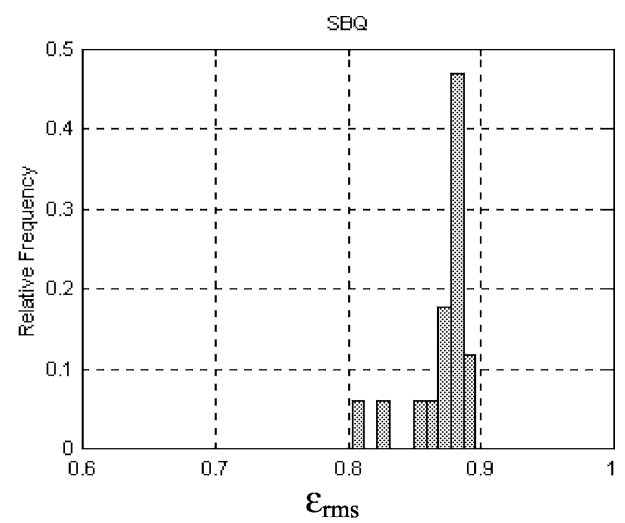

(b)

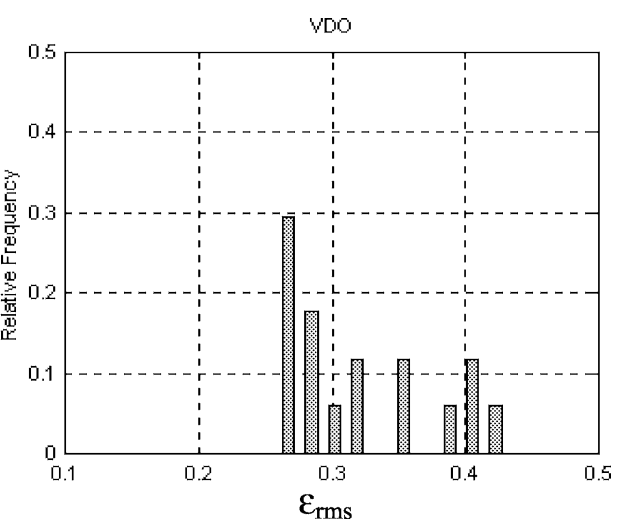

(d)

Fig. 17. Curvature estimation errors for Medioni and Yasumoto's approach with different quantizations: GIQ (a), SBQ (b), scanner (c), and video camera (d). 


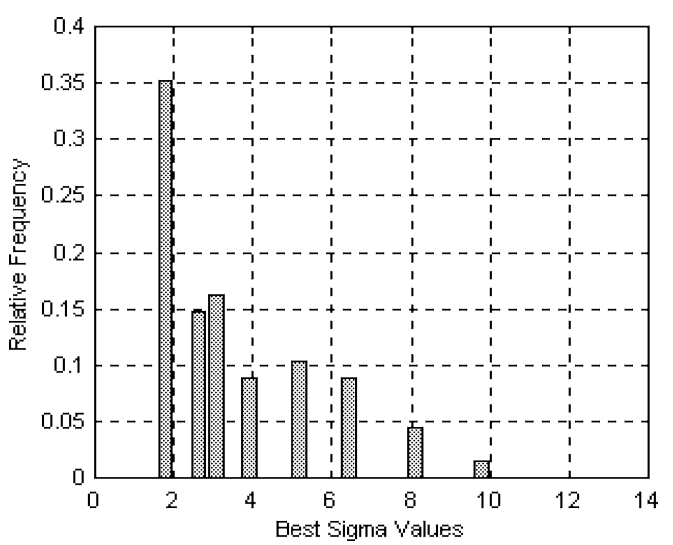

Fig. 18. Histogram of the dispersion of the spatial scale parameter (standard deviation) values for the 1D Fourier-based method (without interpolation).

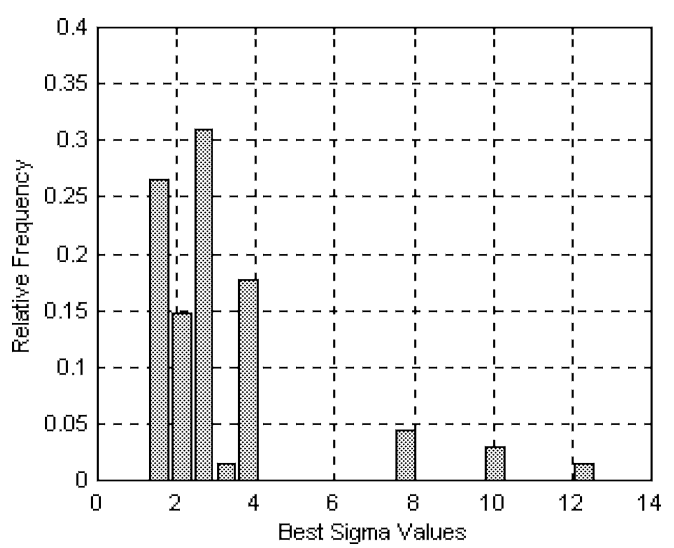

Fig. 19. Histogram of the dispersion of the spatial scale parameter (standard deviation) values for the $2 \mathrm{D}$ Fourier-based method.

portions of the curve that, although close in the $2 \mathrm{D}$ space, are further away along the perimeter.

\section{Discussion}

Regarding the Fourier-based approaches, the first experimental result to be verified (refer to Figs. 12 and 13) indicated no substantial advantage in using different regularizing scale parameters, motivating us to henceforth consider $\sigma=\sigma_{1}=\sigma_{2}$. A particularly important result regarding the Fourier-based methods is the fact that the optimal value of $\sigma$ (implying the smallest error) should be large enough to filter the spatial quantization noise from the contour but still be as small as possible in order not to remove small scale details from the shapes. The optimal values of $\sigma$ have been verified not to vary substantially 


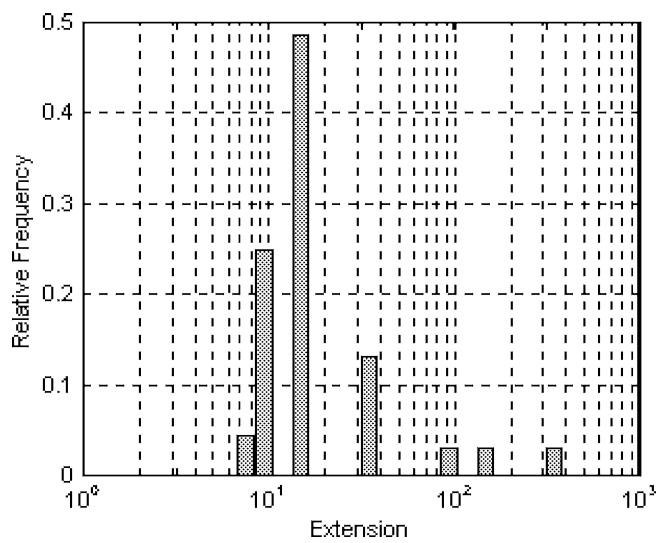

Fig. 20. Histogram of the extension of the spatial scale ensuring error not larger than $10 \%$ of the minimal error, for the 1D Fourier technique.

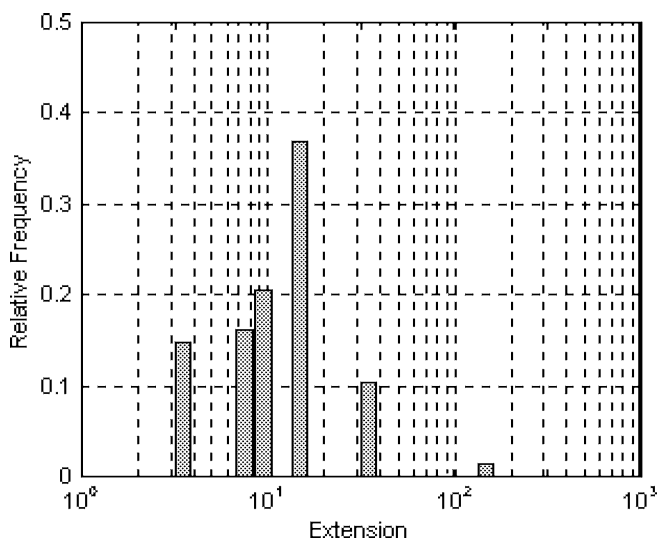

Fig. 21. Histogram of the extension of the spatial scale ensuring error not larger than $10 \%$ of the minimal error, for the $2 \mathrm{D}$ Fourier technique.

between the different classes of curves, which is a desirable feature substantiating the method robustness. As indicated in Figs. 18 and 19, the optimal values of $\sigma$ of more than $70 \%$ of the curves are contained in the interval $[1.5,4]$. Regarding the other considered sensitivity parameter, namely the spatial scale extension (see Fig. 7), about $80 \%$ of all cases processed by the 1D and 2D Fourier techniques (see Figs. 20 and 21) presented an extension larger than 10 pixels, indicating robustness with respect to the choice of $\sigma$.

As indicated by Figs. 14, 15, and 16, the accuracy of the Fourier-based techniques provides estimation errors consistently smaller (by an average factor of 10) than those obtained by Medioni and Yasumoto's approach. On the other hand, the latter technique involves no parameter and is about four times faster than the 1D Fourier technique (see Figs. 8, 9, and 10). The superior accuracy obtained by the Fourier methods has been identified as a consequence of the larger neighborhood of the curve points inherently 
considered in the Fourier approaches. Other general observed tendencies included the fact that the 1D approach considering the linear interpolation outlined in Section 2.2 provided much faster execution time than the 2D method and the 1D approach without interpolation, which is a direct consequence of the use of the fast Fourier transform. On the other hand, similar estimation errors and sensitivity to parametric configurations were obtained for these two techniques (see Fig. 11). It should be also observed that while the speed of the 2D approach depends on the size of the rectangle involving the curve, the execution time of the $1 \mathrm{D}$ approach is defined by the perimeter of the curve. A particularly interesting phenomenon, henceforth called interference, has been observed for the $2 \mathrm{D}$ Fourier approach. As is clear in Fig. 22, curves containing bottlenecks, i.e., portions which are close in the $2 \mathrm{D}$ space but distant along the perimeter, tend to produce error peaks. This has been verified to be a consequence of the fact that the convolution mask comprehends, especially at larger scales, not only the neighborhood of the point where the differential operators are being estimated, but also points from the other portion of the curve. The position of such error peaks is consequently determined by the bottleneck spatial scale. It should be observed that this phenomenon does not imply a shortcoming for curvature estimation, since it occurs at spatial scales much higher than that respective to the optimal error, but can undermine scale space representations derived by the $2 \mathrm{D}$ approach.

Little performance variation has been observed with respect to the three classes of curves and quantization schemes — which indicates that the GIQ and SBQ are good models for the sampling implied by standard scanners and video cameras (see Fig. 11), at least as far as curvature estimation is concerned. An exception has been verified for Medioni and Yasumoto's approach, where the SBQ has implied a much larger error than the other quantizations (Fig. 17), since the SBQ produces more jagged contours (Fig. 5). However, in the case of the Fourier methods, the SBQ often accounted for better accuracy, as a possible consequence of the richer representation of the curve allowed by this quantization scheme.

\section{Concluding remarks}

This article has presented a formal and comprehensive comparative approach to the numerical estimation of curvature by using two Fourier-based approaches (1D and 2D) and the classical method proposed by Medioni and Yasumoto [6], used here in order to provide a comparison standard. Several types of curves, including analytical parametric curves, B-splines, and curves synthesized in the Fourier domain [29], have been considered, and four spatial quantization schemes, namely grid intersect quantization, square box quantization, scanners, and video cameras, have been used in order to produce spatially quantized versions of the considered curves. Special care has been placed on choosing curves ensuring proper representation of the analytical curvature values when represented on the orthogonal lattice, in order to ensure that proper information would be available for the numerical curvature estimation techniques. The performance of the estimation methodologies has been compared according to three principal performance parameters, execution time, curvature estimation errors, and parameter sensitivity (expressed in terms of parameter dispersion and extension), and comprehensive experimental data have been obtained in order to characterize each of the considered curvature estimation techniques. 

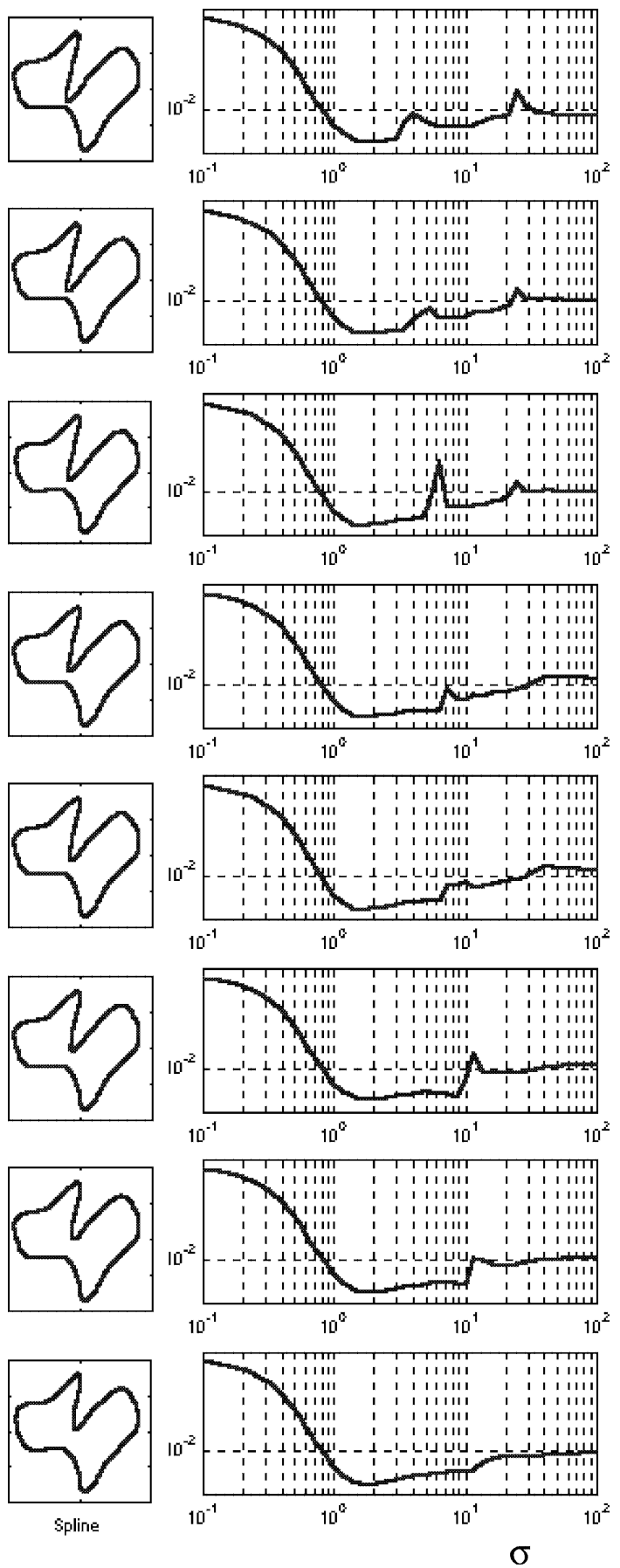

Fig. 22. Illustration of the spatial interference for the 2D Fourier-based approach. 
Generally speaking, Medioni and Yasumoto's approach is particularly suited for situations demanding very fast execution time, but not critical in terms of accuracy. This is in great part a consequence of the fact that this method does not include any resource for filtering out the spatial quantization noise. On the other hand, the Fourier-based schemes account for particularly interesting alternatives for applications requiring very low estimation errors. Moreover, the 1D Fourier approach is faster than the 2D, but the latter has the advantage of allowing the curvature to be estimated not only along the original contour, but throughout its $2 \mathrm{D}$ extension, which can be particularly useful in situations involving curvature estimation for the numerical solution of partial differential equations [14]. The interesting phenomenon of interference has also been observed for the 2D Fourier approach. By characterizing in quantitative terms the advantages and disadvantages of the considered methods, as well as indicating suitable choice of the respective parameters, the obtained results also provide valuable guidelines for those interested in applying the considered techniques in real problems.

\section{Acknowledgments}

Luciano da Fontoura Costa is grateful to FAPESP (Procs 99/12765-2) and CNPq (Procs 301422/92-3 and 468413/00-6) for financial support. Roberto M. Cesar Jr. is grateful to FAPESP for the financial support (98/07722-0), as well as to CNPq (300722/98-2). Andrea Gomes Campos and Luiz Gonzaga Rios-Filho are grateful to FAPESP (Procs 98/12425-4 and 98/13427-0, respectively). Leandro Farias Estrozi is grateful to CAPES.

\section{References}

[1] F. Attneave, Some informational aspects of visual perception, Psych. Rev. 61 (1954) 183-193.

[2] I.T. Young, J.E. Walker, J.E. Bowie, An analysis technique for biological shapes I, Inform. Control 25 (1972) 357-370.

[3] J.E. Bowie, I.T. Young, An analysis technique for biological shapes II, Acta Cytol. 21 (1972) $455-464$.

[4] R.M. Cesar Jr., L. da F. Costa, The application and assessment of multiscale bending energy for morphometric characterization of neural cells, Rev. Sci. Instr. 68 (1997) 2177-2186.

[5] M.E. Morteson, Geometric Modelling, Wiley, New York, 1985.

[6] G. Medioni, Y. Yasumoto, Corner detection and curve representation using cubic B-splines, Comput. Vision Graphics Image Process. 39 (1987) 267-278.

[7] L. da F. Costa, R.M. Cesar Jr., Shape Analysis and Recognition: Theory and Practice, CRC Press, Boca Raton, FL, 2001.

[8] R.M. Cesar Jr., L. da F. Costa, Piecewise linear segmentation of digital contours in $O(N \log (N))$ through a technique based on effective digital curvature estimation, Real-Time Imaging 1 (1995) 409-417, doi:10.1006/rtim.1995.1042..

[9] R.M. Cesar Jr., L. da F. Costa, Towards effective planar shape representation with multiscale digital curvature analysis based on signal processing techniques, Pattern Recog. 29 (1996) 1559-1569.

[10] L. da F. Costa, T.J. Velte, Automatic characterization and classification of ganglion cells from the salamander retina, J. Comp. Neurol. 404 (1999) 33-51.

[11] E. Persoon, K.S. Fu, Shape discrimination using Fourier descriptors, IEEE Trans. Systems Man Cybernet. 7 (1977) 170-179.

[12] M.M.S. Chong, R.K.L. Gay, H.N. Tan, J. Liu, Automatic representation of fingerprints for data compression by B-spline functions, Pattern Recog. 25 (1992) 1199-1210. 
[13] S.A. Mahmoud, Arabic character recognition using Fourier descriptors and character contour encoding, Pattern Recog. 27 (1994) 815-824.

[14] J. Sethian, Level Set Methods: Evolving Interfaces in Geometry, Fluid Mechanics, Computer Vision, and Material Science, 2nd ed., Cambridge University Press, New York, 1999.

[15] D.P. Fairney, P.T. Fairney, On the accuracy of point curvature estimators in a discrete environment, Image Vision Comput. 12 (1994) 259-265.

[16] T. Pavlidis, Algorithms for shape analysis of contours and waveforms, IEEE Trans. Pattern Anal. Mach. Intell. 2 (1980) 301-312.

[17] S. Loncaric, A survey of shape analysis techniques, Pattern Recog. 31 (1998) 983-1001.

[18] S. Marshall, Review of shape coding techniques, Image Vision Comput. 7 (1989) 281-294.

[19] F. Mokhtarian, A. Mackworth, A theory of multiscale, curvature based shape representation for planar curves, IEEE Pattern Anal. Mach. Intell. 14 (1992) 789-805.

[20] L.S. Davis, Understanding shapes: Angles and sides, IEEE Trans. Comput. 26 (1977) 236-242.

[21] C.H. Chen, J.S. Lee, Y.N. Sun, Wavelet transformation for gray-level corner detection, Pattern Recog. 28 (1995) 853-861.

[22] L. Kitchen, A. Rosenfeld, Gray-level corner detection, Pattern Recog. Lett. 1 (1982) 95-102.

[23] R.C. Jain, T.O. Binford, Dialogue: Ignorance, myopia, and naiveté in computer vision systems, Comput. Vision Graphics Image Process. Image Understanding 53 (1991) 112-117.

[24] M. Kunt, Dialogue: Comments on "Dialogue," a series of articles generated by the paper entitled "Ignorance, myopia, and naïveté in computer vision," Vision systems, Comput. Vision Graphics Image Process. Image Understanding 54 (1991) 428-429.

[25] J.L. Barron, D.J. Fleet, S.S. Beauchermin, Performance of optical flow techniques, Int. J. Comput. Vision 12 (1994) 43-77.

[26] K. Cho, P. Meer, J. Cabrera, Performance assessment through bootstrap, IEEE Trans. Pattern Anal. Mach. Intell. 19 (1997) 1185-1198.

[27] D. Demigny, T. Kamlé, A discrete expression of Canny's criteria for step edge detector performances evaluation, IEEE Trans. Pattern Anal. Mach. Intell. 19 (1997) 1199-1211.

[28] M.D. Heath, S. Sarkar, T. Sanocki, K.W. Bowyer, A robust visual method for assessing the relative performance of edge-detection algorithms, IEEE Trans. Pattern Anal. Mach. Intell. 19 (1997) 1338-1359.

[29] C.T. Zahn, R.Z. Roskies, Fourier descriptors for plane closed curves, IEEE Trans. Comput. 21 (1972) 269281.

[30] L.F. Estrozi, A.G. Campos, L.G. Rios, R.M. Cesar Jr., L. da F. Costa, Comparing curvature estimation techniques, in: Proc. 4th Simpósio Brasileiro de Automação Inteligente-SBAI, São Paulo, Brazil, 1999, pp. 58-63.

[31] M. Baroni, G. Barletta, Digital curvature estimation for left ventricular shape analysis, Image Vision Comput. 10 (1992) 485-494.

[32] H. Freeman, Computer processing of line-drawings images, Comput. Surv. 6 (1974) 57-95.

[33] R.F. Gonzalez, R.E. Woods, Digital Image Processing, Addison-Wesley, Reading, MA, 1993.

[34] R. Schalkoff, Digital Image Processing and Computer Vision, Wiley, Singapore, 1989.

[35] J.R. Bennett, J.S. Mac Donald, On the measurement of curvature in a quantized environment, IEEE Trans. Comput. 24 (1975) 803-820.

Leandro Farias Estrozi obtained his B.Sc. in physics from the Institute of Physics at the University of São Paulo, Brazil in 1998, and is currently a Ph.D. student in computational physics at the same institution. His research interests involve 2D and 3D shape analysis, the development of skeletonization algorithms and curvature estimation, psychophysical studies of saccadic vision, the development of tools for scientific visualization, and platforms for WWW-based psychophysical experiments. To find out more about his research and some selected publications take a look at http:// cyvision.if.sc.usp.br/ lfestroz.

Luiz G. Rios-Filho is an aeronautical engineer (Instituto Tecnológico de Aeronaútica, ITA, Brazil). He worked as a researcher at the Instituto Nacional de Pesquisas Espaciais (INPE, Brazil) 
and at aerospace industries. He participated twice in the Brazilian's Antarctic Station Comandante Ferraz (King George Island) for the INPE. He is currently working on his Ph.D. thesis (Instituto de Física de São Carlos, University of São Paulo, Brazil), investigating the shape-function relationship in neural cells using mathematical models of the cells and of the extracellular medium. His research interests are computational neuroscience, neuromorphometry, computational and biological vision, image processing, and pattern recognition.

Andrea Gomes Campos obtained her M.Sc. in computational physics from the Insitute of Physics at the University of São Paulo, Brazil, on the subject of nonlinear edge detection techniques, where she is currently working toward her Ph.D. Her research interests are computer vision, image processing, pattern recognition, computational neuroscience, and neuromorphometry. Her main project is aimed at the characterization, modeling, and computational simulation of neurons considering internal and external factors that can influence their shapes.

Roberto M. Cesar Jr. received a B.Sc. in computer science (UNESP, Brazil), an M.Sc. in electrical engineering (UNICAMP, Brazil), and a Ph.D. in computational physics at the Institute of Physics, University of São Paulo, São Carlos, Brazil, including a period with the Departement de Physique of the Université Catholique de Louvain, Belgium. He held a post-doctoral position at the CVRGSao Carlos in 1997. He is currently a lecturer in the Department of Computer Science of IME-USP. His main research interests concentrate on several problems in the fields of computer vision, pattern recognition, and image processing.

Luciano da F. Costa received a B.Sc. in electronic engineering from University of São Paulo at São Carlos, Brazil, an M.Sc. in applied physics from the Institute of Physics-IFSC, USP-São Carlos, Brazil, and a Ph.D. in electronic engineering from King's College, University of London. $\mathrm{He}$ is an associate professor at the IFSC, where he founded the Cybernetic Vision Research Group in 1993. He is a member of the editorial board of several international journals and has organized several conferences and special issues. His interests include shape analysis, computer vision, signal processing, pattern recognition and datamining, neuroscience, and visual inspection. 